\title{
Association study of childhood obesity with eight genetic variants recently identified by genome-wide association studies
}

\author{
Xiang-Rui Meng'1, Jie-Yun Song ${ }^{1}$, Jun Ma', Fang-Hong Liu', Xiao-Rui Shang ${ }^{1}$, Xu-Jun Guo' and Hai-Jun Wang'
}

BACKGROUND: Being overweight or obese is becoming increasingly common in low- and middle-income countries. The present study aimed to examine association of eight genetic variants with obesity and to estimate the cumulative effects of these variants in Chinese children.

METHODS: We conducted the case-control study in a total of 2,030 subjects. Genotyping of seven novel variants was performed with matrix-assisted laser desorption ionization time of flight mass spectrometry, while rs9939609 was genotyped with tetra-primer amplification refractory mutation system analysis.

RESULTS: The association of two fat mass and obesityassociated gene (FTO) single-nucleotide polymorphisms (SNPs; rs9939609 and rs62048402) with body mass index (BMI) or obesity reached nominal significance at $P<0.05$. We found a cumulative effect of five SNPs on the risk of overweight and obesity (odds ratio $(\mathrm{OR})=1.197,95 \%$ confidence interval $(\mathrm{Cl})$ $=1.068-1.342, P=0.002$ ). Subjects carrying 9 or more effect alleles had a $127 \%$ increased risk of overweight and obesity $(\mathrm{OR}=2.270,95 \% \mathrm{Cl}=1.403-3.671, P=0.001)$ compared with subjects who carry 6 or fewer effect alleles.

CONCLUSION: We confirmed two FTO SNPs (rs62048402 and rs9939609) had nominal significant effects on BMI or obesity. We identified the cumulative effect of five SNPs on risk of overweight and obesity. The results provided evidence for identifying genetic factors related to childhood obesity.

B eing overweight or obese is a significant risk factor for the development of chronic diseases such as type II diabetes and cardiovascular diseases and is becoming increasingly common in low- and middle-income countries (1). Genetic factors contribute to the etiology of obesity and the heritability of body mass index (BMI) ranges 30-70\% (2).

Genome-wide association studies (GWAS) have provided evidences for genetic risk loci for obesity. The largest GWAS of BMI was conducted by the Genetic Investigation of Anthropometric Traits (GIANT) Consortium in 2010 on a sample of 249,796 European individuals (3). The GIANT
Consortium has confirmed 14 known obesity susceptibility loci, and identified 18 new loci associated with BMI $(P<$ $\left.5 \times 10^{-8}\right)$, bringing the total number of such loci to 32 (3). Subsequently, these 32 loci had been studied by many research groups and then replicated in some studies (4-6).

Besides the known 32 loci, we searched published literatures on electronic databases of Pubmed, EBSCO, Web of Science, etc. for novel obesity susceptibility loci, which were identified by GWAS studies. Three such publications were found during 2012-2013. Firstly, in 2012, Ng et al. (7) performed a meta-analysis of two GWAS and the replication in four cohorts of African Americans, revealing five SNPs at four loci (rs6794092 near transmembrane protein 212 (TMEM212), rs268972 near cadherin 12 (CDH12), rs2033195, and rs815611 between microfibrillar-associated protein 3 (MFAP3) and UDP-N-acetyl-alpha-D-galactosamine: polypeptide $\mathrm{N}$-acetylgalactosaminyltransferase 10 (GALNT10), and rs6088887 near Fer-1-like 4 (FER1L4)) associated with BMI $\left(2.4 \times 10^{-6}<P<2.5 \times 10^{-5}\right)$. In the same year, Wang et al. (8) identified a novel locus that influences BMI based on metaanalysis of GWAS data of two Caucasian samples. The strongest associated marker was SNP rs2967951 in rhophilin-associated tail protein 1-like $(R O P N 1 L)$ located at 5p15.2. Moreover, in 2013, Sällman et al. (9) identified SNP rs62048482 in intron 1 of fat mass and obesity-associated gene (FTO) in Swedish children, having stronger association with obesity than SNP rs9939609, although the association between FTO rs9939609 and obesity had been widely confirmed $(10,11)$.

Since the associations between obesity and these novel SNPs recently identified by GWAS have not been validated in independent populations with different ethnicity, it is crucial to explore their effects in Chinese population. So we conducted the present study to examine association of eight gene variants (rs6794092 (PP13439-TMEM212), rs2967951 (ROPN1L), rs268972 (CDH12), rs2033195 (MFAP3-GALNT10), rs815611 (MFAP3-GALNT10), rs62048402 (FTO), rs9939609 (FTO), rs6088887 (FER1L4)) with obesity, and to estimate the cumulative effects of these variants in Chinese children. The study will provide evidences for these SNPs' associations with obesity in 
Table 1. General characteristics of study groups

\begin{tabular}{|c|c|c|c|c|c|c|}
\hline & \multicolumn{2}{|c|}{ ALIR } & \multirow[b]{2}{*}{$P$ value } & \multicolumn{2}{|c|}{ CPOOA } & \multirow[b]{2}{*}{$P$ value } \\
\hline & Normal weight & Overweight and obese & & Normal weight & Overweight and obese & \\
\hline$N$ & 151 & 786 & & 456 & 637 & \\
\hline Age (y) & $14.8 \pm 0.7$ & $14.6 \pm 0.6$ & 0.002 & $11.8 \pm 3.1$ & $11.1 \pm 2.6$ & $<0.001$ \\
\hline $\mathrm{BMI}\left(\mathrm{kg} / \mathrm{m}^{2}\right)$ & $20.4 \pm 1.8$ & $27.5 \pm 3.4$ & $<0.001$ & $18.2 \pm 2.5$ & $24.1 \pm 3.5$ & $<0.001$ \\
\hline
\end{tabular}

ALIR, adolescent lipids, insulin resistance and candidate genes; CPOOA, Comprehensive Prevention project for Overweight and Obese Adolescents.

Table 2. Genotyping information of eight SNPs in Chinese children

\begin{tabular}{|c|c|c|c|c|c|c|c|c|c|c|c|c|c|}
\hline SNP & Chr & Position & Nearest gene & \multicolumn{2}{|c|}{ Allele } & EAF & $\begin{array}{l}\text { EAF in } \\
\text { literature }\end{array}$ & $\begin{array}{l}\text { Call } \\
\text { rate }\end{array}$ & $\begin{array}{c}\text { Total sample } \\
(11 / 12 / 22)\end{array}$ & $\begin{array}{c}\text { HWE } \\
\text { ( } P \text { value })\end{array}$ & $F_{\text {ST }}$ & \multicolumn{2}{|c|}{ Genotype (11/12/22) } \\
\hline rs2967951 & 5 & 10517107 & ROPN1L & $\mathrm{T}$ & $\mathrm{C}$ & 0.67 & 0.10 (ref. 8) & 99.3 & 209/893/914 & 0.297 & 0.343 & $71 / 255 / 277$ & $138 / 638 / 637$ \\
\hline rs268972 & 5 & 22692284 & $\mathrm{CDH} 12$ & $C$ & A & 0.87 & 0.66 (ref. 7) & 99.8 & $28 / 462 / 1534$ & 0.625 & 0.061 & $10 / 146 / 447$ & $18 / 316 / 1087$ \\
\hline rs62048402 & 16 & 52360724 & FTO & A & G & 0.13 & 0.44 (ref. 9) & 99.5 & $38 / 458 / 1523$ & 0.598 & 0.118 & $7 / 128 / 469$ & $31 / 330 / 1054$ \\
\hline rs9939609 & 16 & 52378028 & FTO & A & $\mathrm{T}$ & 0.13 & 0.44 (ref. 9) & 100 & $41 / 462 / 1527$ & 0.670 & 0.118 & $7 / 126 / 474$ & $34 / 336 / 1053$ \\
\hline rs6088887 & 20 & 33634738 & FER1L4 & G & A & 0.91 & 0.79 (ref. 7) & 99.5 & 28/323/1668 & 0.108 & 0.028 & $10 / 104 / 490$ & 18/219/1178 \\
\hline
\end{tabular}

Chr, chromosome; EAF, effect allele frequency; HWE, Hardy-Weinberg equilibrium; Position, NCBI build 36.3 (NCBI, Bethesda, MD); SNP, single-nucleotide polymorphism.

a population whose ethnicity is different from that in original studies.

\section{RESULTS}

The general characteristics of the study groups were shown in Table 1. The study on adolescent lipids, insulin resistance and candidate genes (ALIR) consisted of 151 normal weight children ( 63 females, mean age $14.8 \pm 0.7 \mathrm{y}$, mean BMI $20.4 \pm 1.8 \mathrm{~kg} / \mathrm{m}^{2}$ ) and 786 overweight and obese children ( 267 females, mean age $14.6 \pm 0.6 \mathrm{y}$, mean BMI $\left.27.5 \pm 3.4 \mathrm{~kg} / \mathrm{m}^{2}\right)$. The differences in age and BMI between two groups of ALIR were statistically significant $(P<0.05)$. The Comprehensive Prevention project for Overweight and Obese Adolescents (CPOOA) study group consisted of 456 normal-weight children (260 females mean age $11.8 \pm 3.1 \mathrm{y}$, mean BMI $\left.18.2 \pm 2.5 \mathrm{~kg} / \mathrm{m}^{2}\right)$ and 637 overweight and obese children (222 females, mean age $11.1 \pm 2.6$ $\mathrm{y}$, mean BMI $24.1 \pm 3.5 \mathrm{~kg} / \mathrm{m}^{2}$ ). The differences in sex, age, and BMI between two groups of CPOOA were statistically significant $(P<0.05)$.

The genotype and allele frequencies of all the SNPs were shown in Table 2. Except for the monomorphic SNP rs6794092, the genotypes of the other seven SNPs were in Hardy-Weinberg equilibrium among normal-weight children $(P>0.05)$. F-statisics $\left(F_{S T}\right)$ values between the population of original study and our sample were shown in Table 2, which varied from 0.028 (rs6088887) to 0.343 (rs2967951). Based on the $F_{\mathrm{ST}}$ values, we identified three SNPs having very great or great genetic differentiation, four SNPs moderate differentiation, and only one SNP little differentiation.
Table 3. Association of seven SNPs with BMI in Chinese children

\begin{tabular}{|c|c|c|c|c|c|}
\hline \multirow[b]{2}{*}{ SNP } & \multirow[b]{2}{*}{$\begin{array}{l}\text { Nearest } \\
\text { gene }\end{array}$} & \multicolumn{2}{|c|}{ Our study } & \multicolumn{2}{|c|}{ Discovery study } \\
\hline & & $\beta(S E)$ & $\begin{array}{c}P \\
\text { value }\end{array}$ & $\beta$ (SE) & $\begin{array}{c}P \\
\text { value }\end{array}$ \\
\hline rs2967951 & ROPN1L & $0.187(0.134)$ & 0.162 & 0.76 (ref. 8) & $\begin{array}{l}3.05 \times 10^{-4} \\
\quad \text { (ref. } 8 \text { ) }\end{array}$ \\
\hline rs268972 & $\mathrm{CDH} 12$ & $0.218(0.189)$ & 0.248 & $\begin{array}{c}0.091(0.023) \\
(\text { ref. 7) }\end{array}$ & $\begin{array}{l}5.00 \times 10^{-5} \\
\quad \text { (ref. } 7 \text { ) }\end{array}$ \\
\hline rs2033195 & $\begin{array}{l}\text { MFAP3- } \\
\text { GALNT10 }\end{array}$ & $0.084(0.366)$ & 0.819 & $\begin{array}{c}0.094(0.021) \\
(\text { ref. 7) }\end{array}$ & $\begin{array}{l}5.57 \times 10^{-6} \\
\quad(\text { ref. } 7)\end{array}$ \\
\hline rs815611 & $\begin{array}{l}\text { MFAP3- } \\
\text { GALNT10 }\end{array}$ & $0.024(0.373)$ & 0.948 & $\begin{array}{c}0.095(0.021) \\
(\text { ref. } 7)^{\mathrm{a}}\end{array}$ & $\begin{array}{l}5.36 \times 10^{-6} \\
\quad \text { (ref. } 7 \text { ) }\end{array}$ \\
\hline rs62048402 & FTO & $0.450(0.183)$ & 0.014 & $-{ }^{\mathrm{b}}$ & $-\mathrm{b}$ \\
\hline rs9939609 & FTO & $0.458(0.181)$ & 0.011 & $-\mathrm{b}$ & $-\mathrm{b}$ \\
\hline rs6088887 & FER1L4 & $-0.189(0.208)$ & 0.364 & $\begin{array}{c}0.106(0.025) \\
(\text { ref. 7) }\end{array}$ & $\begin{array}{l}2.53 \times 10^{-5} \\
\quad(\text { ref. } 7)\end{array}$ \\
\hline
\end{tabular}

SNP, single-nucleotide polymorphism.

${ }^{a} \beta$ and SE were reported for the changes in SD unit per copy of the effect allele. ${ }^{b}$ There is no coefficient with BMI in the reference study.

As shown in Table 3, the association of two FTO SNPs (rs62048402 and rs9939609) with BMI reached nominal significance at $P<0.05$. Each effect allele of rs62048402 was associated with increase of $0.450 \mathrm{~kg} / \mathrm{m}^{2}$ in BMI $(P=0.014)$. Each effect allele of rs9939609 was associated with increase of $0.458 \mathrm{~kg} / \mathrm{m}^{2}$ in BMI $(P=0.011)$. As shown in Table 4 , the association of two FTO SNPs (rs62048402 and rs9939609) with obesity also reached nominal significance at $P<0.05$. Each effect allele of rs62048402 was associated with a $25.9 \%$ 
Table 4. Association of seven SNPs with risk of overweight and obesity in Chinese children

\begin{tabular}{|c|c|c|c|c|c|}
\hline \multirow[b]{2}{*}{ SNP } & \multirow[b]{2}{*}{ Nearest gene } & \multicolumn{2}{|c|}{ Our study } & \multicolumn{2}{|c|}{ Discovery study } \\
\hline & & OR $(95 \% \mathrm{Cl})$ & $P$ value & OR $(95 \% \mathrm{Cl})$ & $P$ value \\
\hline rs2967951 & ROPN1L & $1.063(0.912,1.238)$ & 0.435 & $--^{\mathrm{a}}$ & $--^{\mathrm{a}}$ \\
\hline rs2033195 & MFAP3-GALNT10 & $1.316(0.875,1.980)$ & 0.188 & $--^{a}$ & $--^{\mathrm{a}}$ \\
\hline rs815611 & MFAP3-GALNT10 & $1.233(0.810,1.876)$ & 0.329 & $-^{\mathrm{a}}$ & $-^{\mathrm{a}}$ \\
\hline rs9939609 & FTO & $1.322(1.067,1.637)$ & 0.011 & $1.25(1.05-1.48)($ ref. 9$)$ & $<0.012$ (ref. 9) \\
\hline rs6088887 & FER1L4 & $1.162(0.919,1.469)$ & 0.210 & $--^{\mathrm{a}}$ & $-{ }^{\mathrm{a}}$ \\
\hline
\end{tabular}

OR, odds ratio; SNP, single-nucleotide polymorphism.

aThere is no OR value with risk of overweight and obesity in the reference studies.

increased risk of overweight and obesity ( $\mathrm{OR}=1.259,95 \%$ $\mathrm{CI}=1.016-1.560, P=0.035)$. Each effect allele of rs9939609 was associated with a $32.2 \%$ increased risk of overweight and obesity $(\mathrm{OR}=1.322,95 \% \mathrm{CI}=1.067-1.637, P=0.011)$. After randomization tests (100,000 permutations), none of the seven SNPs was significantly associated with BMI or risk of overweight and obesity. We found the effects of the SNPs were in the same direction as those in the discovery studies except for rs6088887 (Tables 3 and 4).

The simple count genetic risk score (GRS), calculated as the effect allele number of 5 SNPs carried by each individual, varied from 2 to 10. As shown in Figure 1, the risk of overweight and obesity increased in a linear model as the simple count GRS increased. On average, each additional effect allele was associated with a $19.7 \%$ increased risk of overweight and obesity $(\mathrm{OR}=1.197,95 \% \mathrm{CI}=1.068-1.342, P=0.002)$. Subjects carrying 9 or more effect alleles had a $127 \%$ increased risk of overweight and obesity ( $\mathrm{OR}=2.270,95 \% \mathrm{CI}=1.403-$ 3.671, $P=0.001)$ compared with subjects that carry 6 or fewer effect alleles. Additionally, OR-weighted GRS value increased the risk of overweight and obesity $(\mathrm{OR}=1.191,95 \% \mathrm{CI}=$ $1.075-1.319, P=0.001)$.

\section{DISCUSSION}

Except for rs9939609, the other seven SNPs were identified in recent $2 \mathrm{y}$. To our knowledge, the present study was the first to validate these SNPs and examine their cumulative effect on BMI or overweight and obesity in other population with different ethnicity.

Recent GWAS studies found novel SNPs associated with BMI and obesity but the proportion of variance explained by individual SNPs is very limited. The aggregation of genetic information, obtained from many markers, into a single GRS variable permits to condense this information into a statistical metric of low dimensionality (12). Li et al. (13) assessed the cumulative effect of 12 SNPs identified by GWAS, and found each additional risk allele being associated with $10.8 \%(P=$ $\left.1.54 \times 10^{-22}\right)$ and $5.5 \%\left(P=3.38 \times 10^{-10}\right)$ increased risks of overweight and obesity. Ntalla et al. (5) assessed cumulative effect of 34 variants in Greek Adolescents, and found a significant association with overweight $(\mathrm{OR}=1.09 ; 95 \% \mathrm{CI}=1.04-1.16$;

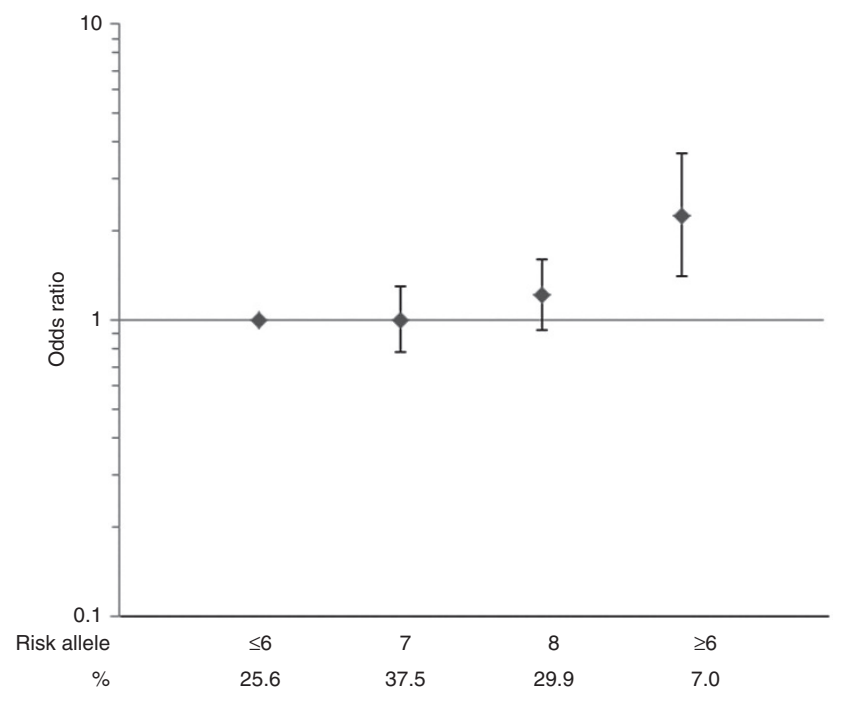

Figure 1. Odds ratios (ORs) and $95 \%$ confidence intervals (Cls) for the risk of overweight and obesity among children with different genetic risk scores. The score $\leq 6$ was used as the reference in logistic regression analysis. On average, each additional effect allele was associated with increased risk of overweight and obesity $(\mathrm{OR}=1.197,95 \% \mathrm{Cl}=1.068-1.342, P=0.002)$.

$P=0.001)$. Similar to these previous studies, we calculated simple count GRS for identifying cumulative effects of genetic variants on overweight or obesity. We found the cumulative effect of five SNPs on overweight and obesity in Chinese children $(\mathrm{OR}=1.197,95 \% \mathrm{CI}=1.068-1.342, P=0.002)$. The OR-weighted GRS had association with overweight and obesity similar to that of simple count GRS $(\mathrm{OR}=1.191,95 \% \mathrm{CI}=$ $1.075-1.319, P=0.001$ ).

The GRS results of our study have also possible biological implications. Previous functional studies provided evidences that six out of eight SNPs in our study have potential effects in the central nervous system (CNS), which had multiple roles on body weight regulation, including appetite, energy expenditure, and other behavioral aspects. GALNT10 with rs2033195 and rs815611 nearby and CDH12 with rs268972 nearby are expressed in CNS (14). Besides, the SNP rs6794092 is located near the gene transmembrane protein 212 (TMEM212) (7), whose family member TMEM18 was found to be associated 
with BMI and expressed at high levels in hypothalamus (15). FTO is highly expressed in hypothalamus, which regulates the energy balance (15). Many studies showed that FTO regulates BMI by influencing energy-dense food intake $(16,17)$. But the functions of two SNPs (ROPN1L and FER1L4) are unknown. The GRS results of our study and the functional evidences suggested further biological studies could study the multigenetic effect and interaction of these genes in a CNS pathway.

We found nominal associations between the two FTO SNPs (rs62048402 and rs9939609) and BMI or risk of overweight and obesity. All the two SNPs were within intron 1 of FTO (15). Sällman et al. (9) found that the intron 1 is the only region within $F T O$ associated with obesity, and reported that rs62048402 $(\mathrm{OR}=1.35,95 \% \mathrm{CI}=1.13-1.60, P<0.0007)$ had a stronger association with obesity than SNP rs9939609 (OR $=1.25,95 \% \mathrm{CI}=1.05-1.48, P<0.012)$. But our study showed that rs62048402 had a lower odds ratio $(\mathrm{OR}=1.259,95 \% \mathrm{CI}$ $=1.016-1.560, P=0.035)$ than $\mathrm{rs} 9939609(\mathrm{OR}=1.322,95 \%$ $\mathrm{CI}=1.067-1.637, P=0.011)$. In Sällman Almén's study (9), rs9939609 and rs62048402 was in a linkage disequilibrium (LD) with $r^{2}$ value of 0.87 among Swedish children. The $r^{2}$ value was 0.96 in our Chinese population, indicating $L D$ of the two FTO SNPs among Chinese population was stronger than that of European population. We considered the difference in association statistics of two SNPs was due to the LD difference.

Despite the fact that the variants had been previously identified to be associated with BMI, we observed that apart from two FTO SNPs, the other six SNPs had no individual effect on BMI or obesity. The unexpected finding might be due to the following reasons. Firstly, there is population differentiation between Chinese ancestry and other populations. The effect allele frequencies of the eight SNPs in the present study were similar to those reported in HapMap Han Chinese, however, different from the frequencies of the original findings in other populations (see Table 2). The effect allele frequency of rs2967951 varied at most, which was 0.67 in our study and 0.10 in Wang's study among Caucasians (8). The SNP rs6794092 was monomorphic in our study, but its effect allele frequency was 0.90 in Ng's study among African American (7). Secondly, the $F_{\mathrm{ST}}$ values of these SNPs varied from 0.028 to 0.343 . We found seven out of eight SNPs having $F_{\mathrm{ST}}$ values over 0.05, indicating moderate to very great genetic differentiation (18). Moreover, heterogeneity of effect size may partly explain the inconsistency in replication. We considered that the effects of these SNPs in Chinese might be lower than that in original populations, which could not be determined with our limited sample size. Further studies should perform fine mapping of this region to clarify the LD difference between different ethnic populations and identify causal variations of obesity.

Different from the discovery studies in adults, our study population was consisted of children and adolescents. Compared with adults, children have higher BMI or obesity heritability and most obese children have simple obesity without complications. Distilling the genetic component of obesity should be easier in children, where environmental exposure and impact had occurred for a relatively short period in their lifetime (19).
So our study in children could help to identify the effects of genetic variations on obesity. In addition, previous studies showed the importance of age-related aspects upon interpretation of GWAS signals. Although the currently known major common variants related to obesity overlap to a substantial degree between children and adults, Scherag et al. identified TNKS/MSRA locus had effects in children and adolescents but no effect in adults (20). We selected the SNPs identified in adults and provided evidences for their effects in children.

The present study had limitations. Firstly, we only selected several novel variants which were recently identified by GWAS to be associated with obesity. We considered the other variants reported before the year 2012 had been studied by many research groups and then replicated in some studies (4-6), some of which in Chinese population (10). But the effects of these novel variants on obesity have not been validated in different populations. So we selected only these variants in this study. However, the cumulative effects or interaction of these SNPs and other known SNPs on pathogenesis of obesity await further studies. The second is the limited sample size and statistic power. Based on the effect estimates from the discovery papers and allele frequencies in the current study population, we calculated the statistic power of our sample for the SNPs. The power for three SNPs was higher than $70 \%$, including FTO rs62048402 (92\%), rs9939609 (73\%) and ROPN1L rs2967951 (99\%). The powers for CDH12 rs268972 and FER1L4 rs6088887 were $62 \%$ and $61 \%$, respectively. But the power was lower for MFAP3-GALNT10 rs2033195 (26\%) and rs815611 (27\%), for minor allele frequency $(0.03)$ is low in the Chinese population. Even in the absence of statistically significant association, the consistency in effect estimates between our study and discovery studies may at least hint at a true association of the SNPs with BMI and obesity in Chinese population, which need further validation studies with large sample size and better power. Thirdly, the case-control study design did not permit us to make conclusions about causality.

In conclusion, we confirmed two SNPs (rs62048402 and rs9939609) in intron 1 of FTO had nominal significant effects on BMI or risk of overweight and obesity. We identified the cumulative effect of five SNPs (rs2967951, rs268972, rs2033195, rs9939609, and rs6088887) on risk of overweight and obesity. The results provided evidences for identifying genetic factors related to childhood obesity.

\section{MATERIALS AND METHODS}

\section{Subjects}

We conducted the case-control study in a total of 2,030 subjects of two independent study groups, including 1,423 overweight or obese cases and 607 normal-weight controls recruited from the urban regions of Beijing, China. The first group came from the study on ALIR. The second study group was from the CPOOA with physical exercise and healthy nutrition as instruments. The ascertainment strategies were very similar in the two studies, except for the age difference $(21,22)$. All the obese individuals in the selected schools were recruited with their voluntary participation. The method of cluster sampling was adopted to recruit non-obese subjects from some classes of each grade in the same schools. The ALIR subjects were ascertained from adolescents aged 14-17 y in 9 middle schools of Dongcheng District of Beijing, including 786 overweight or obese adolescents and 151 
normal-weight adolescents. The CPOOA subjects were recruited from children and adolescents aged 7-18 y in 5 elementary and middle schools of Haidian District of Beijing, comprising 637 overweight or obese children and adolescents and 456 normal-weight children and adolescents. The ascertainment strategies for the two study groups have been previously described in detail previously $(23,24)$. We used the uniform BMI percentile criteria for the participants, which were determined in a representative Chinese population (25). According to criteria, the children and adolescents with an age- and gender-specific BMI $\geq 95$ th percentile are defined as obese; those with an age- and gender-specific BMI between 85th and 95th percentile are defined as overweight. The BMIs of normal-weight students were between the 15 th and 85th percentiles. The individuals with any cardiovascular or metabolic disease were excluded. Two studies were approved by the Ethic committee of Peking University Health Science Center. Written informed consent was provided by all participants and, in the case of minors, their parents.

\section{SNPs Selection and Genotyping}

Firstly, we selected six SNPs associated with BMI or obesity from two new GWAS in $2012(7,8)$. Secondly, the SNP rs62048402 in FTO recently identified by Sälman et al. (9) was included in the present study. Finally, to compare the effects of rs62048402 and rs9939609 (general confirmed to be associated with obesity), rs9939609 was included in the present study.

Genomic DNAs of subjects were extracted from blood leukocytes by the phenol-chloroform extraction method. Genotyping of seven SNPs except for the SNP rs9939609 was conducted on MassARRAY System (Sequenom, San Diego, CA). Primers, including a pair of amplification primers and an extension primer for each SNP, were designed with SpectroDESIGNER software (Sequenom). A multiplex polymerase chain reaction was performed, and unincorporated double-stranded nucleotide triphosphate bases were dephosphorylated with shrimp alkaline phosphatase followed by primer extension. The purified primer extension reaction was spotted on to a 384-element silicon chip (SpectroCHIP, Sequenom) and analyzed in the matrixassisted laser desorption ionization time of flight mass spectrometry (MALDI-TOF MS, Sequenom). The resulting spectra were processed with MassArray Typer (Sequenom). The call rates of the seven SNPs were more than $99.0 \%$.

Genotyping of rs9939609 was carried out with tetra-primer amplification refractory mutation system analysis (tetra-primer ARMS-PCR) (26). The sequences of primers were: $\mathrm{F}_{\text {out }}: 5^{\prime}-$ TGG CTC TTG AAT GAA ATA GGA TTC AGA A-3'; $\mathrm{R}_{\text {out }}: 5^{\text {qut }^{\text {ut }}}$ AGC CTC TCT ACC ATC

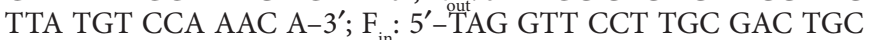
TGT GAA TAT A-3'; $\mathrm{R}_{\text {in }}$ : $5^{\prime}$-GAG TAA CAG AGA CTA TCC AAG TGC ATC TCA-3' (product size: T-allele (321bp, 178bp), A-allele (321bp, 201bp)). Different PCR products were clearly distinguished on $2.5 \%$ agarose gels stained with ethidium bromide. For reference ARMS-PCR of individuals with genotypes identified by sequencing were included in every run. For validity of genotypes, allele assignments were made by at least two experienced individuals independently. Discrepancies were solved unambiguously either by reaching consensus or by repeating. We genotyped $5 \%$ of samples twice for quality control and the genotyping concordance rate was $100 \%$. The call rate of rs9939609 was $100 \%$.

\section{Statistical Analyses}

Statistical analyses were performed using SPSS 18.0 (SPSS, Chicago, IL) and PLINK (Massachusetts General Hospital, Boston, MA). The genotype data of normal-weight group was tested for deviation from Hardy-Weinberg equilibrium. $F$-statistics $\left(F_{\mathrm{ST}}\right)$, a metric representation of the effect of population subdivision, was calculated according to the following formula, $F_{\mathrm{ST}}=\left(\mathrm{P}_{1}-\mathrm{P}_{2}\right)^{2} /\left(\left(\mathrm{P}_{1}+\mathrm{P}_{2}\right)^{\star}\left(2-\left(\mathrm{P}_{1}+\mathrm{P}_{2}\right)\right)\right)$, where $\mathrm{P}_{1}=$ allele frequency in the population of original study and $\mathrm{P}_{2}$ $=$ allele frequency in the population of our study $(27,28)$. A $F_{S T}$ value between 0 and 0.05 indicates little genetic differentiation; a value between 0.05 and 0.15 , moderate differentiation; a value between 0.15 and 0.25 , great differentiation; and values above 0.25 , very great differentiation (18).
The genotypes of each SNP were coded as 0,1 , and 2 according to the number of effect alleles. General linear model was performed to examine the independent effect of each SNP on BMI under additive model with adjustment for age, sex, and study population. Logistic regression model was performed to examine the independent effect of each SNP on risk of overweight and obesity.

To identify cumulative effects of the eight SNPs, we calculated two kinds of GRS, including simple count GRS and OR-weighted GRS. We used Haploview 4.2 (29) to estimate LD between the SNPs. Because rs62048402 was in strong LD with the SNP rs9939609 $\left(r^{2}=\right.$ $0.958)$, and $\mathrm{rs} 2033195$ was in strong LD with $\mathrm{rs} 815611\left(r^{2}=0.956\right)$, rs62048402 and rs815611 were excluded from calculating the GRS. The SNP rs6794092 was also excluded for it was monomorphic in our samples. At first, the simple count GRS was calculated for each individual by adding the number of effect alleles of the remaining five SNPs (rs2967951, rs268972, rs2033195, rs9939609, and rs6088887). Secondly, taking into account the fact that effect sizes among SNPs vary, we calculated an OR-weighted GRS by using weights derived from logging per-allele OR from our study (30). Logistic regression model was used to calculate OR of simple count GRS and OR-weighted GRS for the risk of overweight and obesity.

A two-side $P$ value of $<0.05$ was considered as nominal significance. Empirical significance levels were computed by randomization tests (100,000 permutations) of PLINK (Massachusetts General Hospital). Power calculation was performed using Quanto software (University of Southern California, Los Angeles, CA).

\section{STATEMENT OF FINANCIAL SUPPORT}

The study was supported by grants from National Natural Science Foundation of China (81172683) and the Major State Basic Research and Development Program of China (973 program) (2012CB517501).

Disclosure: The authors declare no conflict of interest.

\section{REFERENCES}

1. Lombard Z, Crowther NJ, van der Merwe L, Pitamber P, Norris SA, Ramsay M. Appetite regulation genes are associated with body mass index in black South African adolescents: a genetic association study. BMJ Open 2012;2:e000873.

2. Herbert A, Gerry NP, McQueen MB, et al. A common genetic variant is associated with adult and childhood obesity. Science 2006;312:279-83.

3. Speliotes EK, Willer CJ, Berndt SI, et al.; MAGIC; Procardis Consortium. Association analyses of 249,796 individuals reveal 18 new loci associated with body mass index. Nat Genet 2010;42:937-48.

4. Qi Q, Chu AY, Kang JH, et al. Sugar-sweetened beverages and genetic risk of obesity. N Engl J Med 2012;367:1387-96.

5. Ntalla I, Panoutsopoulou K, Vlachou P, et al. Replication of established common genetic variants for adult BMI and childhood obesity in Greek adolescents: the TEENAGE study. Ann Hum Genet 2013;77:268-74.

6. Monda KL, Chen GK, Taylor KC, et al.; NABEC Consortium; UKBEC Consortium; BioBank Japan Project; AGEN Consortium. A meta-analysis identifies new loci associated with body mass index in individuals of African ancestry. Nat Genet 2013;45:690-6.

7. Ng MC, Hester JM, Wing MR, et al. Genome-wide association of BMI in African Americans. Obesity (Silver Spring) 2012;20:622-7.

8. Wang KS, Liu X, Zheng S, Zeng M, Pan Y, Callahan K. A novel locus for body mass index on 5p15.2: a meta-analysis of two genome-wide association studies. Gene 2012;500:80-4.

9. Sällman Almén M, Rask-Andersen M, Jacobsson JA, et al. Determination of the obesity-associated gene variants within the entire FTO gene by ultradeep targeted sequencing in obese and lean children. Int J Obes (Lond) 2013;37:424-31.

10. Xi B, Cheng H, Shen Y, et al. Study of 11 BMI-associated loci identified in GWAS for associations with central obesity in the Chinese children. PLoS ONE 2013;8:e56472.

11. da Silva CF, Zandoná MR, Vitolo MR, et al. Association between a frequent variant of the FTO gene and anthropometric phenotypes in Brazilian children. BMC Med Genet 2013;14:34. 
12. Fava C, Sjögren M, Montagnana M, et al. Prediction of blood pressure changes over time and incidence of hypertension by a genetic risk score in Swedes. Hypertension 2013;61:319-26.

13. Li S, Zhao JH, Luan J, et al. Cumulative effects and predictive value of common obesity-susceptibility variants identified by genome-wide association studies. Am J Clin Nutr 2010;91:184-90.

14. Berthoud HR, Morrison C. The brain, appetite, and obesity. Annu Rev Psychol 2008;59:55-92.

15. Willer CJ, Speliotes EK, Loos RJ, et al.; Wellcome Trust Case Control Consortium; Genetic Investigation of ANthropometric Traits Consortium. Six new loci associated with body mass index highlight a neuronal influence on body weight regulation. Nat Genet 2009;41:25-34.

16. Razquin C, Marti A, Martinez JA. Evidences on three relevant obesogenes: MC4R, FTO and PPAR? Approaches for personalized nutrition. Mol Nutr Food Res 2011;55:136-49.

17. Cecil JE, Tavendale R, Watt P, Hetherington MM, Palmer CN. An obesityassociated FTO gene variant and increased energy intake in children. N Engl J Med 2008;359:2558-66.

18. Balloux F, Lugon-Moulin N. The estimation of population differentiation with microsatellite markers. Mol Ecol 2002;11:155-65.

19. Bradfield JP, Taal HR, Timpson NJ, et al.; Early Growth Genetics Consortium. A genome-wide association meta-analysis identifies new childhood obesity loci. Nat Genet 2012;44:526-31.

20. Scherag A, Dina C, Hinney A, et al. Two new Loci for body-weight regulation identified in a joint analysis of genome-wide association studies for early-onset extreme obesity in French and german study groups. PLoS Genet 2010;6:e1000916.
21. Hai-Jun W, Jun M, Zun-Dong Y, Yi-Qun X, Yong-Ping P. Study on the relationship between obesity and metabolic syndrome in adolescents. Chin J Sch Health 2007;28:878-82.

22. Jun M, Shi-Wei Z, Sheng-Xin W, Heng Z, Shan-Shan L. Prevalence of metabolic syndrome among children with different nutritional status in Beijing. Chin J Sch Health 2008;29:103-5.

23. Wang D, Ma J, Zhang S, et al. Association of the MC4R V103I polymorphism with obesity: a Chinese case-control study and meta-analysis in 55,195 individuals. Obesity (Silver Spring) 2010;18:573-9.

24. Wang HJ, Zhang H, Zhang SW, Pan YP, Ma J. Association of the common genetic variant upstream of INSIG2 gene with obesity related phenotypes in Chinese children and adolescents. Biomed Environ Sci 2008;21:528-36.

25. Ji CY; Working Group on Obesity in China. Report on childhood obesity in China (1)-body mass index reference for screening overweight and obesity in Chinese school-age children. Biomed Environ Sci 2005;18:390-400.

26. Ye S, Dhillon S, Ke X, Collins AR, Day IN. An efficient procedure for genotyping single nucleotide polymorphisms. Nucleic Acids Res 2001;29:E888.

27. Weir BS, Cockerham CC. Estimating F-statistics for the analysis of population structure. Evolution 1984;38:1358-1370.

28. Duan S, Zhang W, Cox NJ, Dolan ME. FstSNP-HapMap3: a database of SNPs with high population differentiation for HapMap3. Bioinformation 2008;3:139-41.

29. Barrett JC, Fry B, Maller J, Daly MJ. Haploview: analysis and visualization of LD and haplotype maps. Bioinformatics 2005;21:263-5.

30. Che R, Motsinger-Reif AA. Evaluation of genetic risk score models in the presence of interaction and linkage disequilibrium. Front Genet 2013;4:138. 